\title{
Oral Capecitabine Compared With Intravenous Fluorouracil Plus Leucovorin in Patients With Metastatic Colorectal Cancer
}

\author{
Dayem Uddin ${ }^{1}$, Khan MMR ${ }^{2}$, Shafayat Habib ${ }^{3}$
}

\begin{abstract}
PATIENTS AND METHODS: We prospectively randomized 150 patients to treatment with capecitabine $1,250 \mathrm{mg} / \mathrm{m}^{2}$ administered twice daily days 1 to 14 every 3 weeks, or to the 4weekly Mayo Clinic regimen (5-FU/LV) until disease progression or unacceptable toxicity.

RESULTS: The primary objective, to demonstrate at least equivalent response rates in the two treatment groups, was met. The overall response rate was $18.9 \%$ for capecitabine and $15.0 \%$ for 5-FU/LV. In the capecitabine and 5-FU/LV groups, respectively, median time to disease progression was 5.2 and 4.7 months (log-rank $P=.65$ ); median time to treatment failure was 4.2 and 4.0 months (log-rank $P=.89$ ); and median overall survival was 13.2 and 12.1 months (logrank $P=.33$ ). The toxicity profiles of both treatments were typical of fluoropyrimidines. However, capecitabine led to significantly lower incidences $(P<.00001)$ of stomatitis and alopecia, but a higher incidence of cutaneous hand-foot syndrome $(P<.00001)$. Capecitabine also resulted in lower incidences $(P<.00001)$ of grade $3 / 4$ stomatitis and neutropenia, leading to a lower incidence of grade 3/4 neutropenic fever and sepsis. Only grade 3 hand-foot syndrome $(P<.00001)$ and uncomplicated grade $3 / 4$ hyperbilirubinemia $(P<.0001)$ were reported more frequently with capecitabine.
\end{abstract}

CONCLUSION: Oral capecitabine achieved an at least equivalent efficacy compared with IV 5FU/LV. Capecitabine demonstrated clinically meaningful safety advantages and the convenience of an oral agent.

TAJ 2012; 25: 17-25

\section{Introduction}

Colorectal cancer is the third most commonly diagnosed malignancy and the second leading cause of cancer mortality in Europe and the United States that accounts for an estimated $10 \%$ to $15 \%$ of newly diagnosed cancer cases and approximately 200,000 deaths each year. ${ }^{1-5}$ Earlystage colorectal cancer is localized and resectable, with a 5-year survival rate of $70 \%$ to $80 \% .^{5}$ However, the disease is often asymptomatic in early stages, and up to $30 \%$ of patients present with metastatic disease, which has a 5-year survival rate of $5 \%$ or less. ${ }^{5}$ Overall, approximately $50 \%$ to $60 \%$ of patients eventually develop metastatic or advanced disease.

The fluoropyrimidine fluorouracil (5-FU) is included in essentially all standard and experimental chemotherapy regimens for advanced or metastatic colorectal cancer ${ }^{6-10}$ and results in an increase in survival of approximately

1 Professor and Head, Department of Radiotherapy, Rangpur Medical College, Rangpur.

${ }^{2}$ Associate Professor, Department of Medicine, Rajshahi Medical College, Rajshahi.

${ }^{3}$ M.Phil student, Department of Radiotherapy, Rajshahi Medical College, Rajshahi. 
6 months. ${ }^{3,7}$ Attempts to improve the antitumor efficacy of 5-FU have included biomodulation with agents such as leucovorin, interferon, and Laspartate and schedule modification using protracted or continuous infusion 5-FU regimens. Both of these approaches have led to superior response rates, but survival benefits have been modest ( $<1.5$ months) at best, ${ }^{11,12}$ with several studies and meta-analyses failing to identify any clinically significant survival advantage. ${ }^{13-17}$

Orally administered fluoropyrimidine derivatives have been designed as an alternative approach to optimizing 5-FU-based therapy and have been shown to deliver 5-FU to target cells with predictable kinetics. ${ }^{18,19}$ Oral administration achieves sustained cytotoxic exposure and enables fine control of dosing. In addition, oral agents are more convenient to administer than intravenous (IV) 5-FU, and most patients prefer oral chemotherapy to IV regimens. ${ }^{20-22}$

Capecitabine, an oral fluoropyrimidine carbamate, was rationally designed with the aim of delivering 5-FU predominantly to the tumor cells. ${ }^{23-25}$ Capecitabine is rapidly and extensively absorbed as an intact molecule and is then metabolized to 5FU in three steps. First, it is converted to 5'-deoxy5-fluorocytidine by hepatic carboxylesterase (primarily in the liver), then to 5'-deoxy-5fluorouridine by cytidine deaminase (in tumor cells and liver), and finally to 5-FU by thymidine phosphorylase, which is significantly more active in tumor tissue than in adjacent healthy tissue. ${ }^{24,26}$ Because each step of metabolic conversion occurs with successively greater specificity for tumor cells, capecitabine potentially reduces systemic exposure to 5-FU while maximizing the doseintensity of 5-FU within tumor tissue. Preferential tumor activation of capecitabine has been demonstrated in patients with colorectal cancer. ${ }^{27}$

\section{Patients and Methods Patients}

Patients were eligible for this study if they had advanced or metastatic colorectal cancer and had not received prior chemotherapy for metastatic disease. Adjuvant chemotherapy, if administered, must have been completed at least 6 months before enrollment onto the trial. Histologic or cytologic confirmation of colorectal adenocarcinoma was required, as well as the presence of at least one bidimensionally measurable indicator lesion that had not been irradiated. Ascites and pleural effusion were not considered measurable. One or more indicator lesions were required to be at least $10 \mathrm{~mm}$ in one dimension in the lung or skin or at least $20 \mathrm{~mm}$ in one dimension in the liver or soft tissue masses. Patients had to be at least 18 years of age, ambulatory (Karnofsky performance status $\geq 70 \%$ ), have a life expectancy of at least 3 months, and must have given written informed consent. Contraception was required throughout treatment for females of childbearing potential and for sexually active males. Patients were not included if they were pregnant or lactating, if they were hypersensitive to 5-FU or had previously experienced a severe reaction to fluoropyrimidines, if they had received other experimental drugs within 4 weeks before enrollment, if they had a history of other cancer within the previous 5 years (except for cured basal cell carcinoma of the skin or cervical cancer-insitu), if they had received radiotherapy for their disease within the previous 4 weeks, or had not fully recovered from recent (within 4 weeks) major surgery. Also excluded were patients with organ allografts, CNS involvement of their disease, neurologic or psychiatric disorders that could interfere with treatment compliance, significant cardiac disease or a myocardial infarction within the previous 12 months, serious uncontrolled infections, malabsorption syndrome, or if they lacked physical integrity of their upper gastrointestinal tract. Patients were also not enrolled if screening evaluations revealed significant abnormalities in neutrophils $(<1.5 \times$ $\left.10^{9} / \mathrm{L}\right)$; platelets $\left(<100 \times 10^{9} / \mathrm{L}\right)$; serum creatinine or serum bilirubin (> $1.5 \times$ upper normal limit); ALT; AST; or alkaline phosphatase $(>2.5 \times$ upper normal limit). However, up to five times the upper normal limit for ALT, AST, and alkaline phosphatase was allowed in patients with liver metastases, and up to 10 times upper normal limit for alkaline phosphatase was allowed in patients with bone disease. The study was conducted in accordance with the Declaration of Helsinki and 
all current amendments, and the study protocol was approved by institutional review boards at each participating clinical institution.

\section{Treatment Schedules}

Capecitabine was administered orally twice daily at $1,250 \mathrm{mg} / \mathrm{m}^{2}$ (equivalent to a total dose of 2,500 $\mathrm{mg} / \mathrm{m}^{2} / \mathrm{d}$ ) as an intermittent regimen in 3-week cycles ( 2 weeks of treatment followed by 1 week's rest). For practical reasons, capecitabine doses were rounded to the nearest dose that could be administered with a combination of $500 \mathrm{mg}$ and $150 \mathrm{mg}$ tablets. Capecitabine was given at approximately 12-hour intervals and was taken orally with water within 30 minutes of a meal (breakfast or dinner). The 5-FU/LV was administered according to the Mayo Clinic regimen, which consists of $\mathrm{LV} 20 \mathrm{mg} / \mathrm{m}^{2}$ as a rapid IV injection followed by an IV bolus injection of 5-FU $425 \mathrm{mg} / \mathrm{m}^{2}$, administered daily for 5 days in 4-week cycles. Treatment with capecitabine or 5-FU/LV was continued until the scheduled assessment at 30 weeks or until the development of progressive disease if recorded earlier. In responding patients and those with stable disease, treatment could be continued for up to 48 weeks or for longer at the discretion of the investigator.

\section{Evaluation of Patients}

Assessments of tumor dimensions and involved sites were performed before the start of treatment and were scheduled during therapy after weeks 6 , 12, 18, 24, and 30. Further assessments were performed after weeks 39 and 48 for patients who received prolonged therapy (48 weeks). Follow-up assessments for disease progression and survival monitoring were performed every 3 months after the end of treatment. Tumor dimensions were assessed using computed tomography scans, $\mathrm{x}-$ rays, or magnetic resonance imaging, with tumor response classification based on standard World Health Organization criteria. ${ }^{31}$ Complete response (CR) required the disappearance of all known disease at all involved sites. Partial response (PR) was defined as residual disease with a decrease $\geq$ $50 \%$ in the sum of the products of greatest perpendicular diameters (SPD) of indicator lesions. Progressive disease (PD) was defined as the appearance of a new lesion, or an increase of $25 \%$ in SPD. Stable disease was defined as no change in SPD or a change not corresponding to $\mathrm{CR}$, PR, or PD. To ensure consistency in evaluation, successive tumor measurements and response evaluations for a given patient were conducted by the same investigator. All indicator lesions for evaluation of tumor response were bidimensionally measurable and had not been treated with radiotherapy.

Safety evaluations were conducted at least monthly until 4 weeks after the last administration of therapy, and included assessments of clinical adverse reactions and laboratory parameters. Adverse events were graded on a four-point scale as mild (grade 1), moderate (grade 2), severe (grade 3), or life-threatening (grade 4), as classified by the NCIC CTC grading system (revised December 1994). Hand-foot syndrome (palmar-plantar erythrodysesthesia) was classified as grade 1 (numbness, dysesthesia, painless swelling, or erythema not disrupting normal activities), grade 2 (painful erythema with swelling or affecting daily living activities), or grade 3 (moist desquamation, ulceration, blistering, severe pain, or any symptoms leading to an inability to work or to perform daily living activities).

\section{Statistical Analysis}

The primary end point for this study was the overall objective tumor response rate (CR and PR). The study was designed principally to determine whether capecitabine was at least as active as $5-\mathrm{FU} / \mathrm{LV}$ in the induction of tumor responses. A sample size of 150 patients per treatment group in the all-randomized population was sufficient to achieve $80 \%$ power to demonstrate at least equivalence in overall response rates, assuming a $20 \%$ response rate in both the capecitabine and 5-FU/LV treatment groups, a margin of equivalence of $10 \%$, with alpha equal to 0.025 . The at least equivalence test was based on confidence intervals for the difference in response rates according to HauckAnderson. ${ }^{32}$ In case the at least equivalence test was passed, a further test (two-sided at alpha = 0.05 ) on differences in response rates was 
performed using a $\chi^{2}$ test with Schouten correction. ${ }^{33}$ The study was also powered (80\%) for a difference of $20 \%$ versus $30 \%$ in overall response rate between the two treatment groups.

Secondary efficacy end points were time to response, duration of response, TTP, time to treatment failure, overall survival and quality of life (results to be published separately). Duration of response was calculated according to the World Health Organization response criteria. TTP was calculated from the date of randomization to the first recorded observation of PD or death from any cause. Time to treatment failure included, in addition, premature withdrawals because of adverse events, patients who failed to return, and treatment refusals as events. Overall survival was calculated from the date of randomization to the date of death from any cause.

All analyses of efficacy are reported for the allrandomized population, and all analyses of safety are based on the safety population, which included all patients who received at least one dose of study drug. The IRC assessment was not designed to provide a complete clinical assessment of timerelated end points such as TTP and time to treatment failure. These parameters were analyzed using the investigator assessments, and therefore, for reasons of consistency, the response rate data were also analyzed and reported according to the investigator assessments as well as the IRC assessments.

Adverse reactions, laboratory abnormalities, hospitalizations, and treatment administered for adverse reactions were summarized as incidence rates. For clinically relevant, predefined grade 3/4 adverse reactions typically associated with fluoropyrimidines (diarrhea, stomatitis, hand-foot syndrome, alopecia, nausea, vomiting, and neutropenia) a Kaplan-Meier estimate for the time from randomization to first onset of these adverse reactions was calculated. The log-rank test was used to test for treatment differences.

\section{Results}

\section{Patients and Treatment}

A total of 150 patients were randomized to treatment with capecitabine (75 patients) or 5-
FU/LV (75 patients). Patients were enrolled during a 16-month period from October 2, 2010, to February 4, 2013. Overall, the two treatment groups were well-balanced for all evaluated characteristics. Most patients were elderly, and the colon was the more common site of primary tumor. In both treatment groups, $16 \%$ to $17 \%$ of patients had tumors with poorly differentiated histology. All patients had advanced or metastatic disease, and the most frequently involved metastatic sites were liver (78\%), lung (30\%), and lymph nodes (28\%).

Table 1: Response Rates

\begin{tabular}{|l|l|l|l|l|}
\hline \multirow{2}{*}{ Assessment Response } & \multicolumn{2}{|c|}{$\begin{array}{c}\text { Capecitabine } \\
(\mathbf{n}=\mathbf{7 5})\end{array}$} & \multicolumn{2}{c|}{$\begin{array}{c}\text { 5-FU/LV } \\
(\mathbf{n}=\mathbf{7 5})\end{array}$} \\
\cline { 2 - 5 } & No. & \% & No. & \% \\
\hline IRC & & & & \\
\hline $\begin{array}{l}\text { Overall response, CR or } \\
\text { PR }\end{array}$ & 14 & 18.6 & 11 & 14.66 \\
\hline CR & 1 & 1.33 & 1 & 1.33 \\
\hline PR & 14 & 18.6 & 11 & 14.66 \\
\hline Stable disease & 42 & 56.0 & 42 & 56.0 \\
\hline PD & 9 & 12.0 & 13 & 17.33 \\
\hline Missing post baseline & 8 & 10.6 & 9 & 12.0 \\
\hline
\end{tabular}

*Main reason for missing post baseline: early drop-outs during the first 6 weeks of therapy. These patients were evaluated for the tumor response analysis as nonresponders.

Of the 150 patients enrolled, 149 patients received at least one dose of the allocated treatment drug, approximately $80 \%$ completed 6 weeks of therapy, and approximately $28 \%$ completed the planned treatment period of 30 to 32 weeks. The most frequent reasons for treatment discontinuation were progressive disease (38 patients in the capecitabine group and 41 in the 5-FU/LV group), adverse reactions (10 patients in the capecitabine group and 8 in the 5-FU/LV group), and treatment refusals (5 patients in each group).

Both treatment groups adhered well to the planned dosage regimens. For patients treated with capecitabine, the median dose per cycle was between $82 \%$ and $100 \%$ of that planned. The lower end of median dose per cycle was the result of protocol-specified treatment interruption for toxicity. The median duration of capecitabine treatment was 147 days. For patients treated with 
5-FU/LV, the median dose per cycle of 5-FU was between $95 \%$ and $100 \%$ of that planned. The median duration of treatment was 140 days. For both treatment groups, therefore, actual treatment deviated from planned treatment to only a minor extent and to a similar degree.

\section{Discussion}

This randomized study was designed to compare the efficacy and toxicity of oral capecitabine with that of the Mayo Clinic regimen, IV bolus 5$\mathrm{FU} / \mathrm{LV}$, as first-line treatment in patients with advanced or metastatic colorectal cancer. The results of this trial demonstrate that capecitabine has at least equivalent efficacy compared with IV 5-FU/LV. This study also indicates that capecitabine has clinically meaningful safety advantages over 5-FU/LV.

Baseline disease characteristics, demographic features, treatment history, and prognostic factors were similar between the two treatment groups, and are consistent with those in other studies of colorectal cancer patients who receive first-line 5FU-based chemotherapy. ${ }^{11,13}$ This trial, therefore, provides a sound basis for establishing the activity of capecitabine, and the results seem to be generally applicable to patients with advanced or metastatic colorectal cancer.

Response rates in the all-randomized population based on the IRC assessment were at least equivalent with capecitabine compared with 5FU/LV (Table 1). The response rates observed in the 5-FU/LV group in this study were consistent with those reported for the same 5-FU/LV regimen in other trials, indicating that the patients included in this study were representative of typical populations that receive first-line therapy for metastatic colorectal cancer. ${ }^{16,34-36}$

The toxicity of capecitabine differed in important respects from that of 5-FU/LV. Capecitabine was associated with a substantially lower incidence of the clinically important toxicities, such as severe stomatitis, leucopenia, and neutropenia leading to less neutropenic fever and sepsis compared with 5FU/LV. Although capecitabine and 5-FU/LV both induced toxicities characteristic of fluoropyrimidines, ${ }^{37}$ the onset of typical grade 3 and 4 fluoropyrimidine-related adverse reactions was significantly later and less frequent with capecitabine (Fig 1). Consequently, dose reductions occurred later in patients treated with capecitabine than in those receiving 5-FU/LV. Thus, $27.3 \%$ of the capecitabine patients and $35.1 \%$ of the $5-\mathrm{FU} / \mathrm{LV}$ patients required dose reduction, and median time to dose reduction was 91 days with capecitabine versus 36 days with 5FU/LV. The results of this analysis are clinically relevant because patients who develop progressive disease during the early treatment period will have experienced less drug-related toxicity before discontinuing treatment. Moreover, adverse reactions leading to hospitalization were less frequent in the capecitabine group.

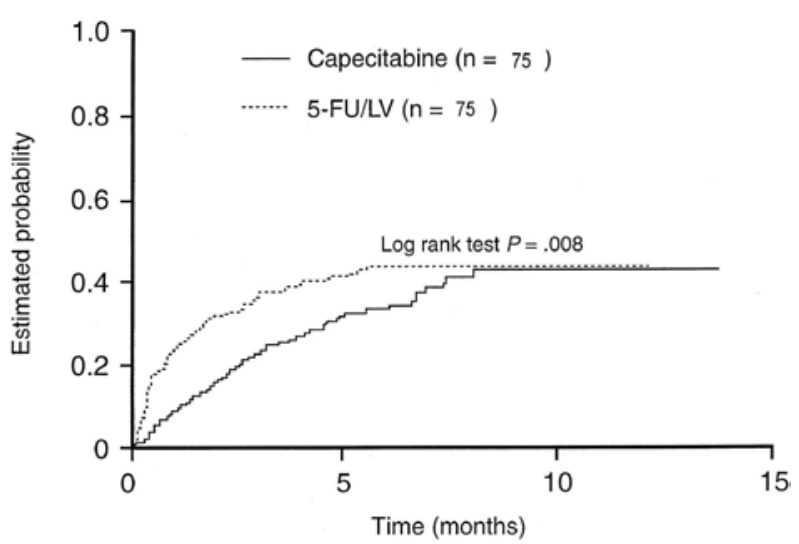

Fig 1. Time to first onset of prespecified treatment-related grade 3 and 4 adverse reactions.

Patients treated with capecitabine who developed hand-foot syndrome responded to dose interruption or reduction according to the protocol and to topical emollients, with improvement and resolution of symptoms. Although $48 \%$ of patients developed some degree of hand-foot syndrome at the planned dose-intensity of capecitabine, only approximately half of those required treatment, which consisted mainly of topical emollients. Only two patients were hospitalized, both for less than 24 hours (one patient had an inpatient visit without overnight stay, and the other had one overnight 
stay for observation only), and fewer than 2\% withdrew from treatment because of this cutaneous reaction. This indicates that hand-foot syndrome did not present a major problem for patients and investigators. In addition, most patients who developed hand-foot syndrome subsequently tolerated treatment with a reduced dose of capecitabine.

Patients in the capecitabine group experienced a higher incidence of NCIC CTC grade 3 or 4 hyperbilirubinemia. However, this hyperbilirubinemia was not clinically significant. Indeed, this level of hyperbilirubinemia corresponds to only grade 2 or 3 bilirubin in the updated and revised NCI CTC criteria. Elevated bilirubin was generally not accompanied by concomitant abnormalities in liver transaminases or alkaline phosphatase, and none of the cases of elevated bilirubin were reported to be irreversible.

Capecitabine therapy in this trial resulted in a significantly superior response rate in patients receiving capecitabine (investigator assessment: 24.8\% $v$ 15.5\% with 5-FU/LV; $P=.005$; IRC assessment: $25.8 \% \vee 11.6 \%$ with $5-\mathrm{FU} / \mathrm{LV} ; P=$ .001 ), and TTP and survival were equivalent for capecitabine and 5-FU/LV.

Capecitabine demonstrated a significantly superior response rate (investigator assessment: 25.5\% $v$ $16.7 \%$ with 5-FU/LV, $P<.00002 ; \quad$ IRC assessment: $22.4 \% \vee 13.2 \%$ with 5 -FU/LV, $P<$ .0001 ) and equivalent TTP and survival. The toxicity profile of capecitabine was similar to that observed in the present study, with a significantly lower incidence of key adverse events (diarrhea, stomatitis, nausea, and alopecia). Hand-foot syndrome was significantly more common with capecitabine but rarely led to treatment withdrawal and resulted in only two brief hospitalizations.

Regimens of 5-FU/LV have been considered standard therapy for patients with advanced colorectal cancer for many years. ${ }^{13,14,40,41}$ The Mayo Clinic regimen is widely used because of its convenience and its greater activity in the induction of response rates compared with bolus
5-FU alone. Response rates reported with the Mayo Clinic regimen range from $9 \%{ }^{41}$ to $43 \%,{ }^{34}$ with median survival typically ranging from 10 to

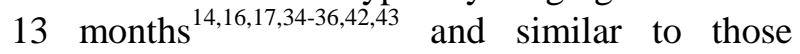
observed with other 5-FU/LV regimens. ${ }^{16,44}$ Administering 5-FU as a prolonged continuous infusion leads to significant increases in response rates compared with bolus 5 -FU alone, ${ }^{17}$ but this has resulted in only a minimal survival benefit ${ }^{11,12}$ or no survival at all. ${ }^{13-17}$ Several studies have demonstrated that an infused regimen of 5-FU/LV (de Gramont or German Association of Medical Oncology regimen) induces a higher response rate compared with IV bolus 5-FU/LV (Mayo Clinic regimen), but no survival difference was observed. ${ }^{14,16}$

Disadvantages of infused 5-FU include inconvenience because of technical requirements, such as central venous access, portable pumps and cost, and the $15 \%$ to $20 \%$ incidence of significant complications, ${ }^{40}$ including infections, bleeding, thrombosis, and pneumothorax, which have a negative impact on quality of life. ${ }^{41}$

Since the start of the present study, other therapeutic agents have demonstrated activity in advanced colorectal cancer either as monotherapy or in combination with 5-FU. Promising results have been obtained with irinotecan in patients with advanced colorectal cancer who have not responded to prior 5-FU therapy ${ }^{45,46}$ and as firstline therapy in combination with $5-\mathrm{FU} / \mathrm{LV}{ }^{42,43}$ The combination of capecitabine plus irinotecan is currently being investigated in several clinical trials. $^{47-49}$ Similarly, the combination of capecitabine plus oxaliplatin is being explored ${ }^{50,51}$ on the basis of reports that oxaliplatin and 5FU/LV may have synergistic activity in patients with advanced colorectal cancer. ${ }^{52}$ On the basis of the results of the present study, we conclude that capecitabine monotherapy provides advantages compared with IV bolus 5-FU/LV in the treatment of advanced colorectal cancer, including a favorable toxicity profile and the convenience of an oral drug that is more attractive to patients, enabling convenient outpatient therapy. 


\section{References}

1. Parkin DM, Pisani P, Ferlay J: Global Cancer Statistics. CA Cancer J Clin 49: 33-64, 1999

2. Greenlee RT, Murray T, Bolden S, et al: Cancer Statistics, 2000. CA Cancer J Clin 50: 7-33, 2000

3. Midgley R, Kerr D: Colorectal cancer. Lancet 353: 391-399, 1999

4. Williams NS, Northover JMA, Arnott SJ, et al: Colorectal tumors, in Peckham $M$, Pinedo $\mathrm{H}$, Veronesi $U$ (eds): Oxford Textbook of Oncology. Oxford, United Kingdom, Oxford University Press, 1995, pp 1133-1168

5. Wingo PA, Ries LA, Parker SL, et al: Long-term cancer patient survival in the United States. Cancer Epidemiol Biomarkers Prev 7: 271-282, 1998

6. Blijham GH: Chemotherapy of colorectal cancer. Anticancer Drugs 2: 233-245, 1991

7. Cunningham D, Findlay $M$ : The chemotherapy of colon cancer can no longer be ignored. Eur J Cancer 29A: 2077-2079, 1993

8. Mayer RJ: Chemotherapy for metastatic colorectal cancer. Cancer 70: 1414-1424, 1992 (suppl)

9. Benson $A B$ III: Therapy for advanced colorectal cancer. Semin Oncol 25: 2-11, 1998 (suppl)

10. Conley BA, Kaplan RS, Arbuck SG: National Cancer Institute Clinical Trials Program in Colorectal Cancer. Cancer Chemother Pharmacol 42: S75-S79, 1998 (suppl)

11. Efficacy of intravenous continuous infusion of fluorouracil compared with bolus administration in advanced colorectal cancer: Meta-analysis Group in Cancer. J Clin Oncol 16:301-308, 1998

12. Thirion P, Wolmark N, Haddad E, et al: Survival impact of chemotherapy in patients with colorectal metastases confined to the liver: A re-analysis of 1458 non-operable patients randomised in 22 trials and 4 meta-analyses: Meta-Analysis Group in Cancer. Ann Oncol 10: 1317-1320, 1999

13. Modulation of fluorouracil by leucovorin in patients with advanced colorectal cancer: Evidence in terms of response rate-Advanced Colorectal Cancer Meta-analysis Project. J Clin Oncol 10:896-903, 1992

14. de Gramont A, Bosset JF, Milan C, et al: Randomized trial comparing monthly low-dose leucovorin and fluorouracil bolus with bimonthly high-dose leucovorin and fluorouracil bolus plus continuous infusion for advanced colorectal cancer. J Clin Oncol 15: 808-815, 1997
15. Leichman CG, Fleming TR, Muggia FM, et al: Phase II study of fluorouracil and its modulation in advanced colorectal cancer: A Southwest Oncology Group study. J Clin Oncol 13: 1303-1311, 1995

16. Schmoll $\mathrm{HJ}$, Köhne $\mathrm{CH}$, Lorenz $\mathrm{M}$, et al: Weekly 24h infusion of high-dose (HD) 5-fluorouracil (5FU24h) with or without folinic acid (FA) vs. bolus 5FU/FA (NCCTG/Mayo) in advanced colorectal cancer (CRC): A randomized phase III study of the EORTC GITCCG and the AIO. Proc Am Soc Clin Oncol 19: 241a, 2000 (abstr 935)

17. Lokich JJ, Ahlgren AJ, Gullo JJ, et al: A prospective randomized comparison of continuous infusion fluorouracil with a conventional bolus schedule in metastatic colorectal carcinoma: A Mid-Atlantic Oncology Program study. J Clin Oncol 7: 425-432, 1989

18. Meropol NJ: Oral fluoropyrimidines in the treatment of colorectal cancer. Eur J Cancer 34: 1509-1513, 1998

19. Pazdur R, Hoff PM, Medgyesy D, et al: The oral fluorouracil prodrugs. Oncology (Huntingt) 12: 4851,1998

20. Payne SA: A study of quality of life in cancer patients receiving palliative chemotherapy. Soc Sci Med 35: 1505-1509, 1992

21. Liu G, Franssen E, Fitch MI, et al: Patient preferences for oral versus intravenous palliative chemotherapy. J Clin Oncol 15: 110-115, 1997

22. Borner $M$, Schöffski $P$, de Wit $R$, et al: $A$ randomized crossover trial comparing oral UFT (uracil/tegafur) + leucovorin (LV) and intravenous fluorouracil $(F U)+$ LV for patient preference and pharmacokinetics in advanced colorectal cancer. Proc Am Soc Clin Oncol 19: 191a, 2000 (abstr 741)

23. Ishikawa $\mathrm{T}$, Utoh $\mathrm{M}$, Sawada $\mathrm{N}$, et al: Tumor selective delivery of 5 -fluorouracil by capecitabine, a new oral fluoropyrimidine carbamate, in human cancer xenografts. Biochem Pharmacol 55: 10911097, 1998

24. Miwa M, Nishida UM, Ishikawa ST, et al: Design of a novel oral fluoropyrimidine carbamate, capecitabine, which generates 5-fluorouracil selectively in tumours by enzymes concentrated in human liver and cancer tissue. Eur J Cancer 34: 1274-1281, 1998

25. Bajetta E, Carnaghi C, Somma L, et al: A pilot safety study of capecitabine, a new oral fluoropyrimidine, in patients with advanced neoplastic disease. Tumori 82: 450-452, 1996 
26. Mackean M, Planting A, Twelves C, et al: Phase I and pharmacologic study of intermittent twice-daily oral therapy with capecitabine in patients with advanced and/or metastatic cancer. J Clin Oncol 16: 2977-2985, 1988

27. Schüller J, Cassidy J, Reigner BG, et al: Tumor selectivity of Xeloda in colorectal cancer patients. Cancer Chemother Pharmacol 45: 291-297, 2000

28. Van Cutsem E, Findlay $M$, Osterwalder B, et al: Capecitabine, an oral fluoropyrimidine carbamate with substantial activity in advanced colorectal cancer: Results of a randomized phase II study. J Clin Oncol 18: 1337-1345, 2000

29. Twelves C, Harper P, Van Cutsem E, et al: A phase III trial (SO14796) of Xeloda (capecitabine) in previously untreated advanced/metastatic colorectal cancer. Proc Am Soc Clin Oncol 18: 263a, 1999 (abstr 1010)

30. Cox JV, Pazdur R, Thibault A, et al: A phase III trial of Xeloda (capecitabine) in previously untreated advanced/metastatic colorectal cancer. Proc Am Soc Clin Oncol 18: 265a, 1999 (abstr 1016)

31. WHO Handbook for Reporting Results of Cancer Treatment. Geneva, Switzerland, World Health Organization, 1979, publication No. 48

32. Hauck WW, Anderson S: A comparison of largesample confidence intervals for the difference of two binomial probabilities. Am Stat 40: 318-322, 1986

33. Schouten HJA, Molenaar IW, van Strick R, et al: Comparing two independent binomial distributions by a modified chi square test. Biometrical Journal 22: 241-248, 1980

34. Poon MA, O'Connell MJ, Moertel CG, et al: Biochemical modulation of fluorouracil: Evidence of significant improvement of survival and quality of life in patients with advanced colorectal carcinoma. J Clin Oncol 7: 1407-1417, 1989

35. Pazdur R, Douillard JY, Skillings JR, et al: Multicenter phase III study of 5-fluorouracil (5-FU) or UFT in combination with leucovorin (LV) in patients with metastatic colorectal cancer. Proc Am Soc Clin Oncol 18: 263a, 1999 (abstr 1009)

36. Carmichael J, Popiela T, Radstone D, et al: Randomized comparative of ORZEL (oral uracil/tegafur (UFT) plus leucovorin (LV)) versus parenteral 5-fluorouracil (5-FU) plus LV in patients with metastatic colorectal cancer. Proc Am Soc Clin Oncol 18: 264a, 1999 (abstr 1015)
37. Meta-Analysis Group in Cancer: Toxicity of fluorouracil in patients with advanced colorectal cancer: Effect of administration schedule and prognostic factors. J Clin Oncol 16:3537-3541, 1998

38. Hoff PM, Ansari R, Batist G, et al: Comparison of oral capecitabine $v$ intravenous 5-fluorouracil plus leucovorin (Mayo Clinic regimen) as first-line treatment in 605 patients with metastatic colorectal cancer: Results of a randomized phase III study. J Clin Oncol 19: 2282-2292, 2001

39. Hoff PM: Capecitabine as first-line treatment for colorectal cancer (CRC): Integrated results of 1207 patients (pts) from 2 randomized, phase III studies. Ann Oncol 11: 60, 2000 (suppl 4, abstr 263)

40. Grem JL: Systemic treatment options in advanced colorectal cancer: Perspectives on combination 5fluorouracil plus leucovorin. Semin Oncol 24: S8S18, 1997 (suppl 18)

41. Schmoll $\mathrm{HJ}$ : Development of treatment for advanced colorectal cancer: Infusional 5-FU and the role of new agents. Eur J Cancer 32A: S18S22, 1996 (suppl 5)

42. Douillard JY, Cunningham D, Roth AD, et al: Irinotecan combined with fluorouracil compared with fluorouracil alone as first-line treatment for metastatic colorectal cancer: A multicentre randomised trial. Lancet 355: 1041-1047, 2000

43. Saltz LB, Cox J, Blanke C, et al: Irinotecan plus fluorouracil and leucovorin for metastatic colorectal cancer. N Engl J Med 343: 905-911, 2000

44. Buroker TR, O'Connell MJ, Wieand HS, et al: Randomized comparison of two schedules of fluorouracil and leucovorin in the treatment of advanced colorectal cancer. J Clin Oncol 12: 14-20, 1994

45. Cunningham D, Glimelius B: A phase III study of irinotecan (CPT-11) versus best supportive care in patients with metastatic colorectal cancer who have failed 5-fluorouracil therapy: V301 Study Group. Semin Oncol 26: 6-12, 1999 (suppl 5)

46. Van Cutsem E, Blijham GH: Irinotecan versus infusional 5-fluorouracil: A phase III study in metastatic colorectal cancer following failure on first-line 5-fluorouracil: V302 Study Group. Semin Oncol 26: 13-20, 1999 (suppl 5)

47. Vanhoefer U, Mayer S, Harstrick A, et al: Phase I study of capecitabine in combination with a weekly schedule of irinotecan (CPT-11) as first-line chemotherapy in metastatic colorectal cancer. Proc Am Soc Clin Oncol 19: 272a, 2000 (abstr 1059) 
48. Vanhoefer U, Mayer S, Achterrath W, et al: Phase I study of capecitabine in combination with a weekly schedule of irinotecan as first-line chemotherapy in metastatic colorectal cancer. Ann Oncol 11: 49, 2000 (suppl 4, abstr 212)

49. Cassata A, Alu M, Beretta E, et al: Capecitabine in combination with two schedules of irinotecan (CPTII) in advanced colorectal cancer: a pilot experience. Ann Oncol 11:S4, 45, 2000 (suppl 4, abstr 192)

50. Díaz-Rubio E, Evans J, Tabernero J, et al: Phase I study of capecitabine in combination with oxaliplatin in patients with advanced or metastatic solid tumors. Proc Am Soc Clin Oncol 19: 198a, 2000 (abstr 772)
51. Evans J, Tabernero J, Cassidy J, et al: Safety profile and preliminary efficacy of capecitabine. Ann Oncol 11: 51, 2000 (suppl 4, abstr 222)

52. Raymond E, Chaney SG, Taamma A, et al: Oxaliplatin: A review of preclinical and clinical studies. Ann Oncol 9: 1053-1071, 1998

53. Dunst J, Reese T, Frings S: Phase I study of capecitabine combined with standard radiotherapy in patients with rectal cancer. Proc Am Soc Clin Oncol 19: 256a, 2000 (abstr 995)

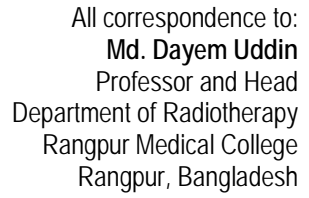

